\title{
Understanding the impact of carrier mobility and mobile ions on perovskite cell performance
}

Nandi Wu, Daniel Walter, Andreas Fell, Kylie Catchpole, Tom White, et al.

Nandi Wu, Daniel Walter, Andreas Fell, Kylie Catchpole, Tom White, Klaus Weber, "Understanding the impact of carrier mobility and mobile ions on perovskite cell performance," Proc. SPIE 10737, Organic, Hybrid, and Perovskite Photovoltaics XIX, 107370T (14 September 2018); doi: $10.1117 / 12.2320223$

Event: SPIE Organic Photonics + Electronics, 2018, San Diego, California, United States 
Invited Paper

\title{
Understanding the impact of carrier mobility and mobile ions on perovskite cell performance
}

\author{
Nandi Wu. *a, Daniel Walter ${ }^{\mathrm{a}}$, Andreas Fell ${ }^{\mathrm{b}, \mathrm{c}}$, Kylie Catchpole ${ }^{\mathrm{a}}$, Tom White ${ }^{\mathrm{a}}$, Klaus Weber ${ }^{\mathrm{a}}$

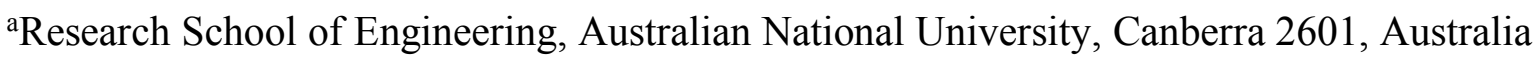 \\ ${ }^{b}$ Fraunhofer Institute for Solar Energy Systems, Freiburg 79110, Germany; ${ }^{\mathrm{c} A F}$ Simulations, \\ Landstr. 12, March 79232, Germany
}

\begin{abstract}
The realization of very high efficiency, stable perovskite solar cells fabricated on a large scale at low cost, has the potential to further lower the cost of photovoltaics. This necessitates an understanding of the properties required of the perovskite material, including the carrier mobility. Perovskite cells also feature mobile ionic species, and the impact of these ions on cell performance - and in particular, to what extent and under what circumstances they may limit device performance - is not well understood. Here, we employ an advanced numerical model that allows for the presence of mobile ionic species to probe the relationship between carrier mobility, the presence of ionic species as well as different possible recombination mechanisms within the cell. We show that a high electron and hole conductivity throughout the device is key to avoiding transport losses. For devices operating significantly below their radiative limit, achieving a sufficiently high conductivity requires high carrier mobilities of at least $10 \mathrm{~cm}^{2} / \mathrm{V}$-s. It is shown that the presence of a single mobile ionic species can lead to effective doping of the perovskite bulk, which is detrimental to cell performance by lowering the conductivity of one type of carrier. The results also indicate that increasing cell VOC closer to its radiative limit is also beneficial for reducing transport losses and pushing cell performance closer to its theoretical limit.
\end{abstract}

Keywords: Perovskite, solar cell, modelling, ionic charge

\section{INTRODUCTION}

Perovskite solar cells (PSCs) have demonstrated high conversion efficiencies in a relatively short period of time and are considered promising candidates as a future, commercially viable photovoltaic technology. Particularly attractive is the combination of perovskite and silicon technologies to develop tandem devices with efficiencies exceeding those possible with a single junction technology. Indeed the first demonstration of such a tandem device with an efficiency exceeding that of the best silicon cell - albeit on a smaller cell area - was recently reported ${ }^{1}$.

Photovoltaic technology has seen dramatic cost reductions in the past two decades, to the point where PV systems are now competitive with, and often cheaper than, fossil fuel based generation in many regions of the world, even without a price on carbon. As a result of the remarkable cost reductions that have been achieved, only a small part of the cost of a PV system - about one quarter - is now contained in the solar cells. The remainder of the cost is therefore in components that will be shared by any other PV technology. For a new technology to be able to outcompete incumbent Si technology, it must therefore achieve almost the same - or preferably a much higher - conversion efficiency as Si technology. Conversion efficiency is key.

*nandi.wu@anu.edu.au

Organic, Hybrid, and Perovskite Photovoltaics XIX, edited by Zakya H. Kafafi, Paul A. Lane, Kwanghee Lee, Proc. of SPIE Vol. 10737, 107370T · ( 2018 SPIE · CCC code: 0277-786X/18/\$18 · doi: 10.1117/12.2320223 
It is therefore important to understand the factors limiting the efficiency of PSCs, and develop avenues to overcome these limitations. PSCs are high voltage devices and as such, theoretically fill factor (FF) values up to $90 \%$ are possible. In practice, much lower values are reported. For example the current record efficiency cell had a FF value of $80.1 \%{ }^{2}$. Fill factor is therefore one area where further significant efficiency improvements may be obtained. In order to do so, it is necessary to understand the factors limiting it.

Solar cell current-voltage (I-V) characteristics are often described using the Shockley equation, which provides a convenient tool of analysing cell characteristics and extracting key parameters:

$$
J=J_{0}\left[\exp \left(V / n_{i d} V_{T}\right)-1\right]-J_{g e n}
$$

where $\mathrm{J}$ is the current density, $\mathrm{V}$ is the terminal voltage, $\mathrm{J}_{0}$ is the dark saturation current density, $\mathrm{J}_{\text {gen }}$ is the photogenerated current density, $\mathrm{V}_{\mathrm{T}}$ is the thermal voltage and $\mathrm{n}_{\text {id }}$ is the ideality factor. The above equation can be easily extended to include lumped, ohmic series and shunt resistances. In this form, it is often applied to analyse cell IV characteristics and determine the values of the lumped parameters, which are then assigned to various physical regions of the device (such as series resistance of metal fingers, etc.).

However, the Shockley equation is only valid under certain conditions as it assumes that there exist no sources that may result in a nonlinear series or shunt resistance. One important example of a nonlinear resistance arises when significant carrier transport limitations exist within the cell. For example, Würfel et al. ${ }^{3}$ showed that for organic cells, the Shockley equation cannot be applied since the low carrier mobilities in the absorber result in a cell response that is transport dominated. In such a case, cell I-V characteristics often do not conform to those obtained from the extended Shockley equation. Even when they do, the interpretation of the characteristics using the extended Shockley equation results in erroneous values for the extracted lumped parameters. In organic solar cells at least, the limited carrier mobilities substantially reduce the device efficiency, and they do so primarily through a reduction in the cell's fill factor.

Mobilities in the absorbers of PSCs are usually significantly higher than those in organic cells, with typical reported values in the range $1-10 \mathrm{~cm}^{2} / \mathrm{V}-\mathrm{s}{ }^{4,5}$ so it may be that the mobilities typically encountered should not result in a significant deviation of the cell behaviour from that predicted by the Shockley equation, and that mobility does not limit fill factor and cell efficiency - at least for cells with well aligned charge extraction layers. On the other hand, PSCs are known to contain mobile ionic charges which influence cell behaviour. The impact of these mobile charges on cell performance, through their interaction with other material properties such as carrier mobility, is not well understood.

In this paper, we model the behaviour of cells with and without mobile ionic charge in order to better understand the impact of carrier mobility and mobile ionic species on cell performance. Given the complexity of PSCs and the very large parameter space, the goal is not obtain definitive answers but to obtain some insights into how the various material properties can interact to influence cell performance.

\section{MODELLING APPROACH}

We model the cells using the "one-dimensional detailed solver" of solar cell simulation software Quokka 3. ${ }^{6}$ Quokka3 models the charge-carrier transport in the perovskite absorber using the semiconductor drift-diffusion models used to model inorganic semiconductors. The solution domain consists only of the perovskite absorber layer. The electron and hole transport layers (ETLs and HTLs) are treated as metal-semiconductor boundaries with a defined barrier and the interfaces are specified solely through specification of the transport layer work functions and the interface recombination velocity $\mathrm{S}$ such that

$$
U_{S}=S \cdot \Delta n
$$

where $\mathrm{U}_{\mathrm{S}}$ is the recombination rate and $\Delta n$ is the excess carrier density at the interface. Bulk recombination is modelled as a combination of radiative and trap-mediated recombination. Quokka enables implementation of the general as well as simplified Shockley-Read-Hall (SRH) formalism to simulate trap-mediated recombination ${ }^{7}$. The simplified formalism is only valid in situations where the trap density is an order of magnitude lower than the charge carrier density. Here, we employ the simplified SRH formalism a for the simulations of this work, in order to limit the complexity of the model.

In the simplified SRH formalism, the bulk defect-assisted recombination rate is expressed as 


$$
U_{S R H}=\frac{n p-n_{i}^{2}}{\tau_{p 0}\left(n-n_{1}\right)+\tau_{n 0}\left(p-p_{1}\right)}
$$

with

$$
\tau_{p 0} \equiv \frac{1}{\sigma_{p} v_{t h} N_{T}} ; \tau_{n 0} \equiv \frac{1}{\sigma_{n} v_{t h} N_{T}}
$$

and $\mathrm{n}_{1}, \mathrm{p}_{1}$ being statistical factors given by

$$
n_{1} \equiv N_{C} \exp \left(\frac{E_{T}-E_{C}}{k T}\right) ; p_{1} \equiv N_{V} \exp \left(\frac{E_{V}-E_{T}}{k T}\right)
$$

where $\mathrm{V}_{\text {th }}$ is the thermal velocity of charge carriers, $\mathrm{N}_{\mathrm{T}}$ is the trap density, $\sigma_{p}$ and $\sigma_{n}$ are the defect capture cross sections for holes and electrons, and $\mathrm{E}_{\mathrm{T}}, \mathrm{E}_{\mathrm{C}}$ and $\mathrm{EV}_{\mathrm{V}}$ are the trap, conduction band edge and valence band edge energy levels, respectively. The trap (defect) energy level plays a critical role in determining the recombination dynamics of a particular defect.

The one dimensional detailed solver in Quokka enables the simulation of mobile ions through the modelling of drift diffusion for two additional charge carriers: anions and cations. It is assumed that ions are neither generated nor "recombine". Instead, a fixed and equal, average concentration of anions and cations migrate through the perovskite bulk via drift and diffusion forces. The solver computes the ionic-electronic Debye layer at the transport-layer interfaces, without the need for simplifications such as fixed accumulation layers ${ }^{8}$ or asymptotic approximations. ${ }^{10}$ The interfaces are impermeable to ion flow and do not sink or source ions to the bulk.

In the model, the ions act as dopants but not as recombination sites. Since they act as dopants, the accumulation or depletion of these ions alters the local doping in the cell, which in turn can impact recombination through the SRH formalism. More specifically, altered doping in any region of the device will alter the electron and hole populations, which directly appear as terms in the SRH rate equation, therefore leading to altered recombination rates. In addition, and more significantly, changes in local doping and local electron / hole populations can result in altered barriers to carrier transport.

The above model represents, in a sense, the simplest possible way of accounting for the impact of mobile ionic charge. For example, it neglects the possibility that mobile ions may themselves act as recombination centres. It is not possible at this stage to be certain that this interpretation of ionic influence is correct. We note, however, that the application of a such a simple model - and also the use of Quokka - has been very successful in predicting many of the complex cell behaviours observed. ${ }^{8,9}$

Photon recycling is not implemented in the solver. For our simulations, we employ the solver in steady-state mode, where all carriers distributions (ions, electrons and holes) are computed at their steady-state conditions at each bias point. It other words, we model the true steady-state IV curves. It should be noted that such steady state I-V sweeps are never done in practice since they are often impractical. This point must be kept in mind when examining the simulation outputs and comparing them against reported results.

More details of regarding Quokka3, including the extensive validation of the solver, can be found in the publication of Walter et al. ${ }^{9}$ Table 1 summarises the key model parameters, where the values for $\mathrm{N}_{\mathrm{c}}, \mathrm{N}_{\mathrm{v}}, \mathrm{E}_{\mathrm{g}}, \varepsilon_{\mathrm{r}}$ and $\mathrm{B}$ were taken from Kirchartz et al. ${ }^{11,12}$ 
Table 1. Summary of material parameters.

\begin{tabular}{|l|l|l|}
\hline Parameter & Symbol & Value \\
\hline Perovskite absorber thickness & & $500 \mathrm{~nm}$ \\
\hline Electron affinity & $\mathrm{EEA}_{\mathrm{E}}$ & $3.7 \mathrm{eV}$ \\
\hline Conduction band density of states & $\mathrm{N}_{\mathrm{c}}$ & $2 \times 10^{18}$ \\
\hline Valance band density of states & $\mathrm{N}_{\mathrm{v}}$ & $2 \times 10^{18}$ \\
\hline Band gap & $\mathrm{E}_{\mathrm{g}}$ & $1.6 \mathrm{eV}$ \\
\hline Relative permittivity & $\varepsilon_{\mathrm{r}}$ & 30 \\
\hline Radiative recombination coefficient & $\mathrm{B}$ & $6 \times 10^{-10} \mathrm{~cm}^{3} \mathrm{~s}^{-1}$ \\
\hline Work function - electron transport layer & $\mathrm{W}_{\mathrm{F}, \mathrm{ETL}}$ & $3.7 \mathrm{eV}$ \\
\hline Work function - hole transport layer & $\mathrm{W}_{\mathrm{F}, \mathrm{HTL}}$ & $5.3 \mathrm{eV}$ \\
\hline Illumination profile & & $\mathrm{AM} 1.5 \mathrm{G}$ \\
\hline Cation Concentration & $\mathrm{N}_{\mathrm{an}}$ & $5 \times 10^{18}$ \\
\hline Anion Concentration & $\mathrm{N}_{\mathrm{cat}}$ & $5 \times 10^{18}$ \\
\hline
\end{tabular}

\section{RESULTS}

\subsection{Absorber with high mobility and no mobile ions}

Reduction in cell FF may arise due to recombination dynamics or due to transport limitations. In the absence of any transport limitations and any shunts, a cell's IV response can be well characterised by the Shockley equation (eq. 1). From this, the expression for FF can be obtained:

$$
F F=\frac{v_{o c}-\ln \left(v_{o c}+0.72\right)}{v_{o c}+1}
$$

with $v_{O C} \equiv V_{O C} / n_{i d} V_{T}$

where $\mathrm{n}_{\mathrm{id}}$ is the ideality factor. In practice, a cell cannot be characterised by a single value for the ideality factor. Nevertheless, this simplified picture is sufficient for our present discussion.

Different recombination mechanisms can be associated with different values of the ideality factor and therefore impose different limitations on FF. In our models we consider only radiative and trap-assisted recombination. Radiative recombination results in an ideality factor of 1 . For trap-assisted or SRH recombination, the situation is not as simple as is sometimes portrayed. The ideality factor arising from SRH recombination varies between 1 and 2 and depends on both the nature of the defects and the carrier concentrations in the different regions of the cell, as will be borne out by the modelling results.

With only radiative and SRH recombination, we can therefore expect an ideality factor between 1 and 2, and a FF (in the absence of other parasitic mechanisms) between $83 \%(\mathrm{n}=2)$ and $90 \%(\mathrm{n}=1)$.

In our model, no shunt resistances are included. The only source of what can be defined as a series resistance is due to transport limitations in the absorber itself. These arise from the fact that the movement of electron and hole currents within the cell and out the contacts requires a driving force. This is apparent from the equations relating the current flows to the quasi-Fermi levels (qFLs):

$$
J_{n}(x)=q \cdot \mu_{e} \cdot n(x) \cdot \frac{d E_{F n}}{d x} \quad J_{p}(x)=q \cdot \mu_{h} \cdot p(x) \cdot \frac{d E_{F p}}{d x}
$$


These expressions show that significant current flows require either a high conductivity (high $\mu_{e} n$ or $\mu_{p} p$ product) or a change in the quasi-Fermi levels - the latter being indicative of a resistive loss in the device since it results in an internal voltage (internal qFL separation) that is greater than the external measured voltage.

Given the two fundamentally different sources of FF reduction, it is helpful to attempt to understand these separately before considering situations where both may occur. We therefore first consider the simplest case of a PSC with a high mobility of $100 \mathrm{~cm}^{2} / \mathrm{V}$-s and with no mobile ions in the bulk. The absorber is undoped. The high mobility is intended to ensure a high electron and hole conductivity everywhere in the absorber, which should result in minimal resistive losses.

We consider 4 recombination scenarios:

1. Radiative recombination only

2. Interface recombination only, characterised by surface recombination velocity S. S has the same value at both perovskite interfaces

3. Bulk SRH recombination only, trough deep (mid-gap) trap states

4. Bulk SRH recombination only, through shallow trap-states [include trap parameters for all cases in the table above]

To facilitate a comparison of the different scenarios, we adjust the defect densities for scenarios 2-4 to obtain the same cell $V_{O C}$. This $V_{O C}$ is chosen to be $120 \mathrm{mV}$ below the Voc of the cell with radiative recombination only. The resulting $V_{O C}$ is representative of typical, very high quality PSCs as reported in the literature. Such a drop in Voc means that in these scenarios the cells are dominated by trap-assisted recombination, with radiative recombination accounting for only $\sim 1 \%$ of all recombination events.

Table 2 summarises the cell parameters obtained for all four scenarios. For the cell with radiative recombination only, the cell performs at its theoretical limit, with the exception that $V_{O C}$ is underestimated due to the absence of photon recycling in our model. The IV parameters are in good agreement with those of Kirchartz ${ }^{12}$ for the case where photon recycling was not considered.

Table 2. Simulated cell performance with different recombination scenarios.

\begin{tabular}{|l|l|l|l|l|}
\hline \multicolumn{1}{|c|}{ Parameter } & \multicolumn{1}{c|}{$\begin{array}{c}\text { Radiative } \\
\text { recombination }\end{array}$} & $\begin{array}{c}\text { Surface } \\
\text { recombination }\end{array}$ & $\begin{array}{c}\text { Shallow bulk } \\
\text { recombination }\end{array}$ & $\begin{array}{c}\text { Deep bulk } \\
\text { recombination }\end{array}$ \\
\hline $\mathrm{JSC}\left(\mathrm{mA} / \mathrm{cm}^{2}\right)$ & 24.1 & 23.5 & 24.1 & 24.1 \\
\hline $\mathrm{VOC}(\mathrm{V})$ & 1.249 & 1.129 & 1.129 & 1.129 \\
\hline $\mathrm{FF}(\%)$ & 90 & 87.2 & 88.9 & 84.1 \\
\hline Efficiency (\%) & 27.1 & 23.1 & 24.2 & 22.9 \\
\hline
\end{tabular}

For the cells with surface recombination or with shallow bulk traps, we observe, in addition to the reduction in $V_{\text {oc, }}$ a slight drop in FF. This reduction in FF is due to transport limitations (even at the high mobility chosen for the simulations) as can be easily shown by further increasing the mobility, which results in FF values approaching $90 \%$.

In the case of deep bulk traps, on the other hand, FF is limited chiefly by the recombination dynamics. Fig. 1 shows the band diagram for the cell with deep bulk traps at MPP as well as the corresponding electron and hole concentration distributions, together with the recombination rate. These devices - since they are undoped and have no mobile ionic species - effectively feature a wide space-charge region where the $n \sim p$. Under these conditions, and with deep traps, the SRH equation predicts an ideality factor of 2 , which is borne out in the high recombination rate near the centre of the absorber layer. 
(a)

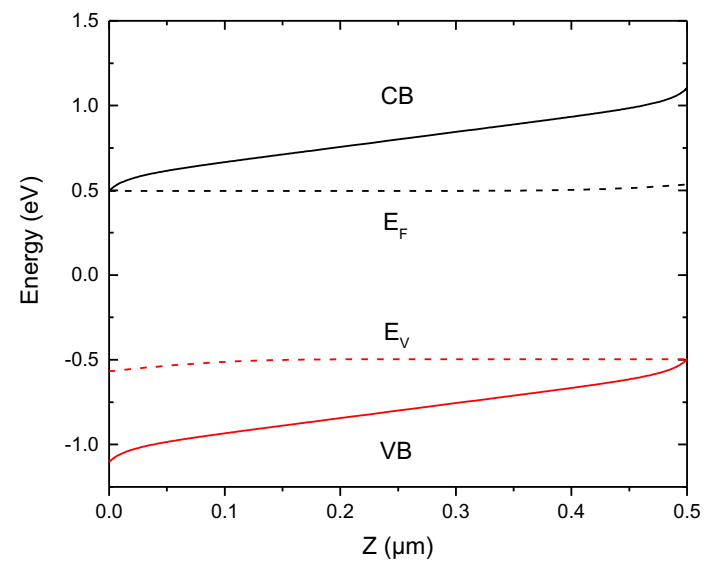

(b)

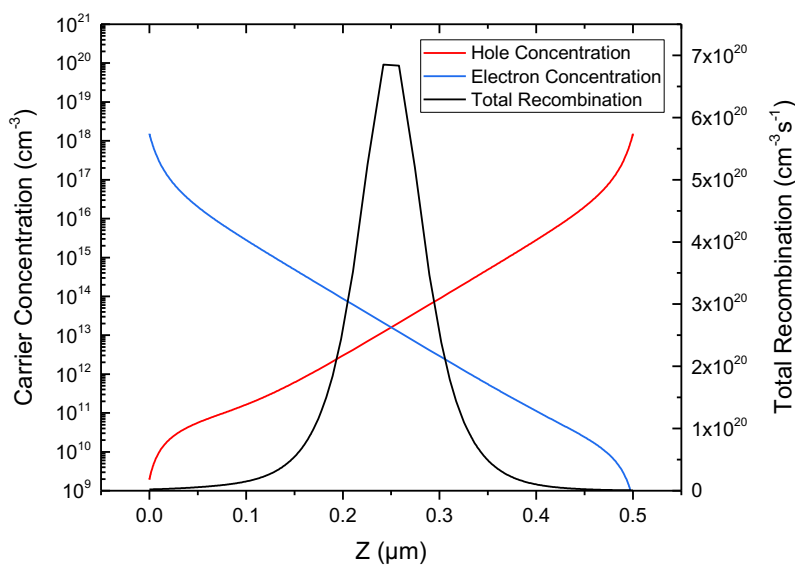

Figure 1. (a) Band diagram of simulated cell with deep bulk recombination at maximum power point. (b) carrier concentrations and total recombination rate across the perovskite absorber of the same cell at maximum power point.

In the case of the cell with surface recombination, since the majority carrier concentration is always much higher than the minority carrier concentration at either contact due to the (idealised) nature of the contacts, this condition results (from the $\mathrm{SRH}$ equation) in $\mathrm{n}=1$. On the other hand, in the case of shallow bulk traps, the trap energy level means that the relative carrier concentrations are not important in determining the value of the ideality factor, and $n=1$.

In practice, cells will feature a combination of surface and bulk recombination, where the bulk traps may be shallow or deep. It would therefore be expected that the cell ideality in the absence of transport limitations will be between 1 and 2, and that recombination dynamics will limit the achievable FF in any given material to a value between 84 and $90 \%$, as we have observed on the basis of photoluminescence measurements. ${ }^{13}$

\subsection{Impact of mobile ions}

Next, we introduce mobile ionic species into our model. We introduce either mobile cations, or both mobile cations and anions, at a concentration of $5 \times 10^{18} \mathrm{~cm}^{-3}$, which is similar to reported values mobile ion concentrations for $\mathrm{MAPbI}_{3}{ }^{14-16}$ The results of density functional theory calculations by Eames et al. ${ }^{10}$ suggest that both the $\mathrm{I}^{-}$anion and the $\mathrm{MA}^{+}$cation may be mobile in $\mathrm{MAPbI}_{3}$. The ionic mobility is set to $10^{-12} \mathrm{~cm}^{2} \mathrm{~V}^{-1} \mathrm{~s}^{-1}$ for mobile species. It should be noted that since the steady-state response is simulated, the value of the ion mobility has no impact on the result. Further, when two mobile ionic species are simulated, different values of the ionic mobility for the different species produce identical results to a simulation using the same mobility value for both species.

As in the previous section, we adjust the defect parameters for each of the recombination scenarios 2-4 above in order to achieve the same Voc of $1.129 \mathrm{~V}$.

The addition of mobile ions is found to have no significant impact on the I-V characteristics of cells operating at the radiative limit (recombination scenario 1). In contrast, when parasitic recombination pathways dominate, the presence of mobile ionic species can have a significant impact. Fig. 2(a) shows the I-V curves of the cells with no mobile ions, with mobile cations or with both mobile cations and anions, for the case of shallow bulk traps while figs. 2(b-d) shows selected band diagrams at MPP and short circuit conditions.

Next, we introduce mobile ionic species into our model. We introduce either mobile cations, or both mobile cations and anions, at a concentration of $5 \times 10^{18} \mathrm{~cm}^{-3}$, which is similar to reported values mobile ion concentrations for $\mathrm{MAPbI}_{3}{ }^{14-16}$ The results of density functional theory calculations by Eames et al. ${ }^{10}$ suggest that both the $\mathrm{I}^{-}$anion and the $\mathrm{MA}^{+}$cation may be mobile in $\mathrm{MAPbI}_{3}$. The ionic mobility is set to $10^{-12} \mathrm{~cm}^{2} \mathrm{~V}^{-1} \mathrm{~s}^{-1}$ for mobile species. It should be noted that since the steady-state response is simulated, the value of the ion mobility has no impact on the result. Further, when two mobile 
ionic species are simulated, different values of the ionic mobility for the different species produce identical results to a simulation using the same mobility value for both species.

As in the previous section, we adjust the defect parameters for each of the recombination scenarios 2-4 above in order to achieve the same $\mathrm{V}_{\mathrm{OC}}$ of $1.129 \mathrm{~V}$.

The addition of mobile ions is found to have no significant impact on the I-V characteristics of cells operating at the radiative limit (recombination scenario 1). In contrast, when parasitic recombination pathways dominate, the presence of mobile ionic species can have a significant impact. Fig. 2(a) shows the I-V curves of the cells with no mobile ions, with mobile cations or with both mobile cations and anions, for the case of shallow bulk traps while figs. 2(b-d) shows selected band diagrams at MPP and short circuit conditions.

The above conclusions were found to broadly hold regardless of the recombination mechanism in the cell (recombination via surface traps, shallow bulk or deep bulk traps), with a significant reduction in $\mathrm{JSC}_{\mathrm{SC}}$ in all cases, together with some reduction in FF. The details vary from case to case. In measurements typically performed on laboratory cells, where cells are swept in the reverse and then in the forward directions at different sweep rates, such behavior would likely manifest itself as significant hysteresis in the IV curves, as well as a significantly lower steady-state power output compared to the MPP output determined from a typical IV curve (usually performed with a relatively fast sweep rate).

Thus, our simulations suggest that the presence of single mobile ionic species can be detrimental to device performance, resulting in both a reduced (steady-state) JSC as well as FF. A significant performance reduction can occur even if the carrier mobilities are very high. The magnitude of the impact would depend on the concentration of the mobile ionic species as well as the energy alignment at the contacts. In our modelling, only rather ideal contacts are considered. Our results also suggest that doping of the perovskite film is more likely to be detrimental than beneficial, as it results in asymmetrical carrier transport and can contribute to the formation of a transport barrier. A detrimental impact of doping on cell performance has also been predicted by Kirchartz et al. ${ }^{11}$, albeit for cells operating at the radiative limit where the impact only observed at lower mobility values.

\subsection{Impact of finite carrier mobility}

The results presented above were all obtained with high electron and hole mobilities of $100 \mathrm{~cm}^{2} / \mathrm{V}$-s. Actual mobility values in perovskite thin films are likely to be significantly lower. To explore the impact of lower mobility values on device performance, we repeat selected simulations over a larger range of mobilities. For simplicity, electron and hole mobilities are kept the same. In contrast to the simulation results presented above, here we no longer constrain the Voc to $1.129 \mathrm{~V}$. The starting point for all the simulations are the parameters that were used in the previous sections, which resulted in a $\mathrm{V}_{\mathrm{OC}}$ of $1.129 \mathrm{~V}$ when the mobility was set to 100 for both electrons and holes. 
(a)

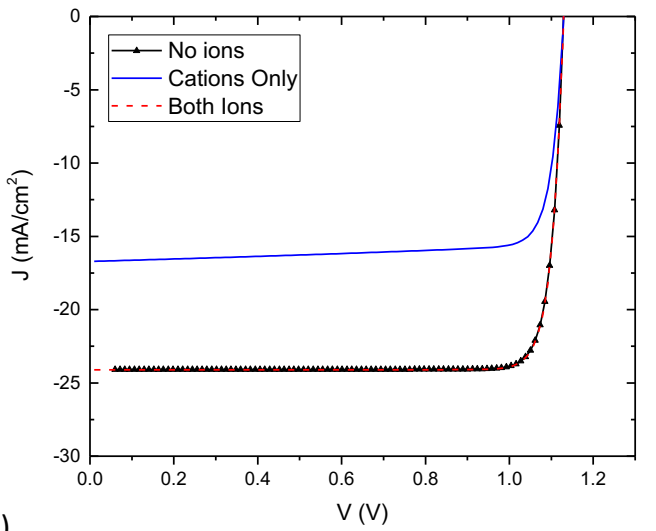

(c)
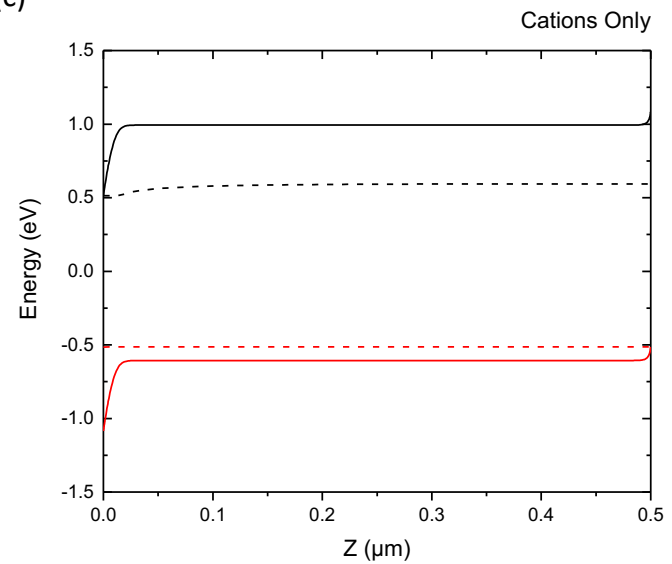

(e)

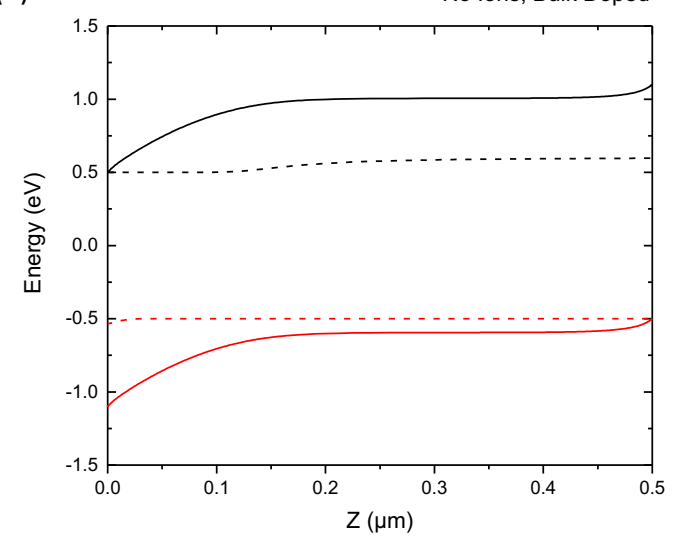

(b)

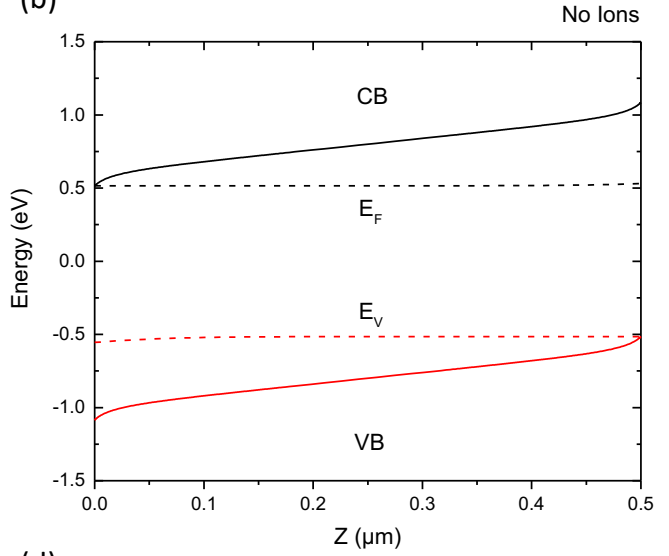

(d)
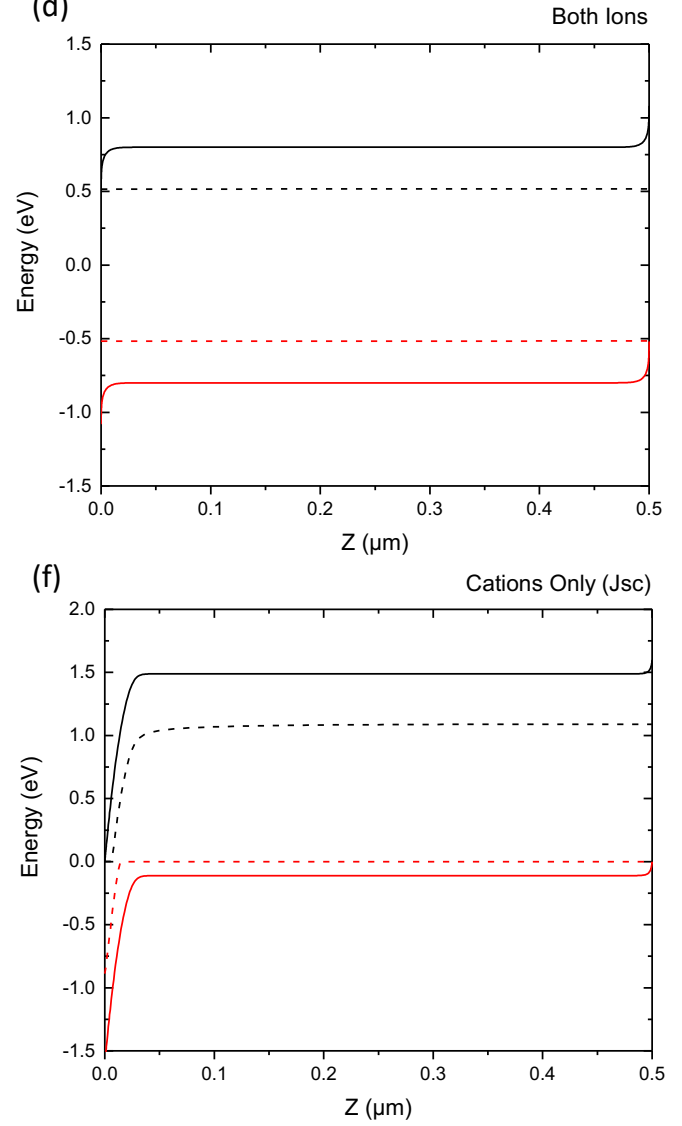

Figure 2. (a) Simulated IV curves of perovskite cells with no mobile ions, only cations, and both cations and anions. Cells here have matched to the same $\mathrm{V}_{\mathrm{OC}}$ of $1.129 \mathrm{~V}$. (b)-(d) Band diagrams of the perovskite bulk at maximum power point with no ions, only cations, and both anions and cations. (e) Band diagram of perovskite absorber at maximum power point without ions, but is p-type doped with the same concentration as excess cations in Figure 2(c). (f) Band diagram of the absorber with only mobile cations at $\mathrm{J}_{\mathrm{SC}}$.

Fig. 3 shows the dependence of cell efficiency on mobility for the four recombination scenarios, in the absence of mobile ionic species. In the case of the cell operating at the radiative limit, we see no significant reduction in performance until the mobility drops below $1 \mathrm{~cm}^{2} / \mathrm{V}-\mathrm{s}$, in broad agreement with the results of Kirchartz et al. When non-radiative 
recombination is introduced, the cell becomes more sensitive to mobility and a significant dropoff in cell efficiency is observed below $10 \mathrm{~cm}^{2} / \mathrm{V}$-s when the bulk recombination dominates. Again, the chief reason for the reduction in cell performance is the reduced conductivity in the cell, which induces drops in the qFLs near the contacts. Cells become more sensitive to carrier mobility when additional recombination pathways are introduced because cells with parasitic recombination operate at lower voltages, which translates to lower electron and hole concentrations and therefore lower conductivities.

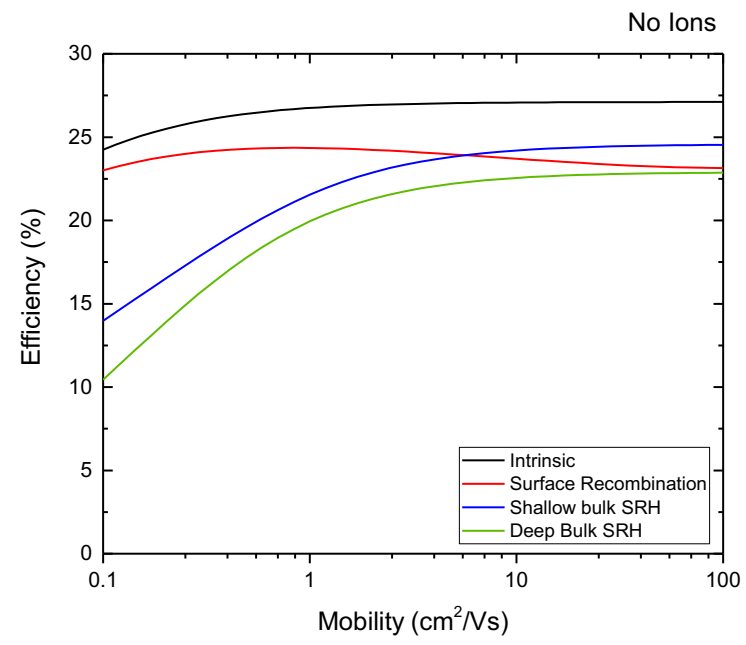

Figure 3. The effect of carrier mobility on perovskite cell efficiency with different recombination scenarios with no mobile ions.

The cell that is dominated by surface recombination shows a different trend, with a slight increase in efficiency as the mobility is reduced. For this cell, there are two competing effects of mobility. Since recombination is limited to the surfaces, a lower mobility reduces the flow of minority carriers to the surface where they can recombine, increasing cell Voc. This increase in cell voltage is also beneficial for the electron and hole conductivity. On the other hand, the reduction in mobility acts to reduce carrier conductivity which results in an increased transport barrier. These effects more or less cancel each other out over a broad range of mobilities, since in these simulations we no longer constrain Voc.

Consistent with our observations for high mobility, the behaviour of cells with two mobile ionic species of equal concentration was found to be similar to that of cells with no mobile ions. We attribute this to the similar concentrations of electrons and holes in the device, and hence the similar conductivities.

Fig. 4 shows the variation of cell efficiency with mobility for the case where mobile cations are present. Not surprisingly, a reduction in mobility below $100 \mathrm{~cm}^{2} / \mathrm{V}$-s results in a further decrease in cell performance in all cases, but with the detailed trend dependent on the recombination mechanism. Even the cell operating at the radiative limit is significantly affected once carrier mobility drops significantly below $100 \mathrm{~cm}^{2} / \mathrm{V}$-s. Given that the primary reason for this performance drop is the effective doping of the device, which causes a reduction in the concentration and conductivity of one type of carrier (electrons in this case), this reinforces the conclusion that doping of the perovskite film may have a detrimental impact on cell performance. 


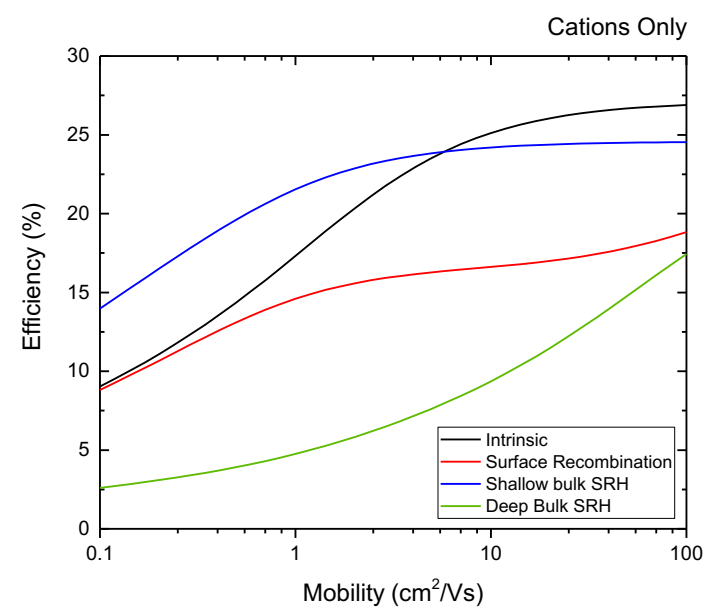

Figure 4. The effect of carrier mobility on perovskite cell efficiency with different recombination scenarios with mobile cations.

\section{CONCLUSIONS}

We have examined, through the use of an advanced device model, the impact of carrier mobility, the presence of mobile ionic species and recombination on the performance of PSCs. The modelling results show that poor carrier transport may limit cell performance under a range of circumstances, and that the impact can manifest itself as a loss in steady-state JSC or FF, or both. Factors that increase transport losses include low carrier densities in the cell at maximum power point or $V_{O C}$, as well as significant doping in the absorber. The presence of a single mobile ionic species is shown to be detrimental to device performance chiefly because such a mobile species may result in effective doping of the absorber bulk. On the other hand, the presence of two ionic species of equal concentrations but opposite polarity (representing, for example, the mobile MA+ and I- vacancies in MAPI) do not have a significant impact on cell performance, despite the fact that they result in a near complete screening of the electric field in the bulk of the cell. Further, the relative mobilities of the ionic species, while having a major impact on the cell transient response, do not impact the steady state device performance.

Finally, our results suggest that both high carrier mobilities of at least $10 \mathrm{~cm}^{2} / \mathrm{V}$-s as well as high Voc (low parasitic losses) are highly beneficial to minimise the impact of cell transport losses and realise highly efficient perovskite devices. 


\section{REFERENCES}

[1] OxfordPV, 25 June 2018, <https://www.oxfordpv.com/news/oxford-pv-sets-world-record-perovskite-solar-cell >

[2] W. S. Yang, B. W. Park, E. H. Jung, N. J. Jeon, Y. C. Kim, D. U. Lee, S. S. Shin, J. Seo, E. K. Kim, J. H. Noh, and S. Il Seok, "Iodide management in formamidinium-lead-halide-based perovskite layers for efficient solar cells," Science (80-. )., vol. 356, no. 6345, pp. 1376-1379, 2017.

[3] U. Würfel, D. Neher, A. Spies, and S. Albrecht, "Impact of charge transport on current-voltage characteristics and power-conversion efficiency of organic solar cells," Nat. Commun., vol. 6, p. 6951, 2015.

[4] I. Grill, M. F. Aygüler, T. Bein, P. Docampo, N. F. Hartmann, M. Handloser, and A. Hartschuh, "Charge transport limitations in perovskite solar cells: The effect of charge extraction layers," ACS Appl. Mater. Interfaces, p. acsami.7b09567, 2017.

[5] C. S. Ponseca, T. J. Savenije, M. Abdellah, K. Zheng, A. Yartsev, T. Pascher, T. Harlang, P. Chabera, T. Pullerits, A. Stepanov, J.-P. Wolf, and V. Sundström, "Organometal halide perovskite solar cell materials rationalized: ultrafast charge generation, high and microsecond-long balanced mobilities, and slow recombination.," J. Am. Chem. Soc., vol. 136, no. 14, pp. 5189-92, Apr. 2014.

[6] A. Fell, D. Walter, and S. Glunz, "A Fast and Easy Perovskite Solar Cell Simulation Tool Featuring Ion Migration," in 33rd European Photovoltaic Solar Energy Conference and Exhibition, 2017, pp. 26-31.

[7] W. Shockley and W. T. Read, "Statistics of the Recombinations of Holes and Electrons," Phys. Rev. B, vol. 87, pp. $835-842,1952$.

[8] D. Jacobs, Y. Wu, H. Shen, C. Barugkin, F. Beck, T. White, K. Weber, and K. Catchpole, "Hysteresis phenomena in perovskite solar cells: the many and varied effects of ionic accumulation," Phys. Chem. Chem. Phys., vol. 19, pp. 3094-3103, 2017.

[9] D. Walter, A. Fell, Y. Wu, T. Duong, C. Barugkin, N. Wu, T. P. White, and K. Weber, "Transient Photovoltage in Perovskite Solar Cells: Interaction of Trap-Mediated Recombination and Migration of Multiple Ionic Species," $J$. Phys. Chem. C, vol. 122, pp. 11270-11281, 2018.

[10] G. Richardson, S. E. J. O'Kane, R. G. Niemann, T. A. Peltola, J. M. Foster, P. J. Cameron, and A. B. Walker, “Can slow-moving ions explain hysteresis in the current-voltage curves of perovskite solar cells?," Energy Environ. Sci., vol. 9, no. 4, pp. 1476-1485, 2016.

[11] T. Kirchartz and U. Rau, "Decreasing Radiative Recombination Coe ffi cients via an Indirect Band Gap in Lead Halide Perovskites," 2017.

[12] T. Kirchartz, F. Staub, and U. Rau, "Impact of Photon Recycling on the Open- Circuit Voltage of Metal Halide Perovskite Solar Cells," 2016.

[13] N. Wu, Y. Wu, D. Walter, H. Shen, T. Duong, D. Grant, C. Barugkin, X. Fu, J. Peng, T. White, K. Catchpole, and K. Weber, "Identifying the Cause of Voltage and Fill Factor Losses in Perovskite Solar Cells by Using Luminescence Measurements," Energy Technol., vol. 5, no. 10, pp. 1827-1835, 2017.

[14] S. D. Stranks, V. M. Burlakov, T. Leijtens, J. M. Ball, A. Goriely, and H. J. Snaith, "Recombination Kinetics in Organic-Inorganic Perovskites: Excitons, Free Charge, and Subgap States," Phys. Rev. Appl., vol. 2, no. 3, p. 34007, Sep. 2014.

[15] G. Xing, N. Mathews, S. S. Lim, N. Yantara, X. Liu, D. Sabba, M. Grätzel, S. Mhaisalkar, and T. C. Sum, "Lowtemperature solution-processed wavelength-tunable perovskites for lasing," vol. 13, no. May, pp. 476-480, 2014.

[16] C. Eames, J. M. Frost, P. R. F. Barnes, B. C. O'Regan, A. Walsh, and M. S. Islam, "Ionic transport in hybrid lead iodide perovskite solar cells," Nat. Commun., vol. 6, p. 7497, Jun. 2015.

[17] T. Kirchartz, J. Bisquert, I. Mora-Sero, and G. Garcia-Belmonte, "Classification of solar cells according to mechanisms of charge separation and charge collection," Phys. Chem. Chem. Phys., vol. 17, no. 6, pp. 4007-4014, 2015. 